\title{
Formal [3 + 2] and [3 + 3] Additions of Acceptor-Substituted Cyclopropylmethylsilanes to Allenylsilanes
}

\author{
Veejendra K. Yadav* and Vardhineedi Sriramurthy \\ Department of Chemistry, Indian Institute of Technology, Kanpur 208 016, India \\ e-mail: vijendra@iitk.ac.in
}

\section{SUPPLEMENTARY INFORMATION}

Experimental details and characterization data (14 pages). 
General. ${ }^{1} \mathrm{H}$ and ${ }^{13} \mathrm{C}$ spectra were recorded on JEOL JNM-LA400 instrument using solutions in $\mathrm{CDCl}_{3}$. The ${ }^{1} \mathrm{H}$ and ${ }^{13} \mathrm{C}$ spectra are referred, respectively, to TMS used as an internal standard and the central line for $\mathrm{CDCl}_{3}$ respectively. Elemental $(\mathrm{C}$ and $\mathrm{H})$ analyses were performed on a Perkin-Elmer 240-C automatic elemental analyzer. All the reactions were carried out using freshly distilled dry solvents. Column chromatography was performed over silica gel (100-200 mesh) obtained from Acme Chemicals using mixtures of hexanes and EtOAc. The separation of isomers was achieved by radial chromatography using plates coated with silica gel $60 \mathrm{PF}_{254}$ (E-Merck). Solvents were removed under reduced pressure on a rotovap. Organic extracts were dried with anhydrous $\mathrm{Na}_{2} \mathrm{SO}_{4}$.

Materials. The allenylsilane 2a was purchased from Sigma-Aldrich and the allenylsilane 2b was prepared according to a literature procedure. ${ }^{1}$

\section{General procedure for cyclopropanation of allyl tert-butyldiphenylsilane with diazo} compounds. The solution of a diazo compound $(2 \mathrm{mmol})$ in $\mathrm{CHCl}_{3}(2 \mathrm{~mL})$ was added to a stirred solution of allyl tert-butyldiphenylsilane $(1.120 \mathrm{~g}$, $4 \mathrm{mmol})$ and $\mathrm{Rh}_{2}(\mathrm{OAc})_{4}(43 \mathrm{mg}$, $0.01 \mathrm{mmol})$ in anhydrous $\mathrm{CHCl}_{3}(0.5 \mathrm{~mL})$ under nitrogen over $10 \mathrm{~h}$ using a syringe pump. The reaction mixture was stirred further for $5 \mathrm{~h}$ and the solvent was removed. Purification of the residue by silica gel column chromatography (EtOAc/hexanes) furnished a mixture of the cis- and trans-products. The separation of the cis- and trans-isomers was achieved by radial chromatography (EtOAc/hexanes).

1a. Following the general procedure outlined above, cyclopropanation of allyl tertbutyldiphenylsilane with phenyl diazomethyl ketone afforded 1a (trans/cis $=2.4: 1)$ as a colorless liquid, $597 \mathrm{mg}, 75 \%$.

trans-1a. ${ }^{1} \mathrm{H}$ NMR (400 MHz, $\left.\mathrm{CDCl}_{3}\right): \delta$ 7.71-7.69 (2H, m), 7.61-7.55 (4H, m), 7.51-7.47 $(1 \mathrm{H}, \mathrm{m}), 7.38-7.23(8 \mathrm{H}, \mathrm{m}), 2.31-2.27(1 \mathrm{H}, \mathrm{m}), 1.67-1.58(1 \mathrm{H}, \mathrm{m}), 1.45-1.39(1 \mathrm{H}, \mathrm{dd}, J=$ 15.0, $6.5 \mathrm{~Hz}), 1.37-1.31(2 \mathrm{H}, \mathrm{m}), 1.05(9 \mathrm{H}, \mathrm{s}), 0.78-0.73(1 \mathrm{H}, \mathrm{m}) .{ }^{13} \mathrm{C}$ NMR $(100 \mathrm{MHz}$, $\left.\mathrm{CDCl}_{3}\right): \delta 199.3,137.6,135.8,135.7,134.2,134.0,132.1,129.0,128.9,128.0,127.7,127.4$, 27.7, 27.3, 23.2, 21.7, 17.8, 15.6. Anal Calcd for $\mathrm{C}_{27} \mathrm{H}_{30} \mathrm{OSi}$ : C, 81.35; H, 7.59. Found: C, $81.22 ; \mathrm{H}, 7.45$. 
cis-1a. ${ }^{1} \mathrm{H}$ NMR (400 MHz, $\left.\mathrm{CDCl}_{3}\right)$ : $\delta$ 7.94-7.91 (2H, m), 7.69-7.67 (2H, m), 7.69-7.28 (11H, m), 2.67-2.62 (1H, m), 1.60-1.50 (1H, m), 1.42-1.35 (1H, dd, $J=15.1,9.8 \mathrm{~Hz}), 1.31-1.26$ $(1 \mathrm{H}, \mathrm{dd}, J=15.1,3.9 \mathrm{~Hz}), 1.23-1.17(1 \mathrm{H}, \mathrm{m}), 1.02(9 \mathrm{H}, \mathrm{s}), 0.95-0.90(1 \mathrm{H}, \mathrm{dt}, J=7.8,4.4$ Hz). ${ }^{13} \mathrm{C}$ NMR (100 MHz, $\left.\mathrm{CDCl}_{3}\right): \delta 198.8,139.0,136.1,135.9,134.6,134.5,132.3,129.0$, $128.9,128.3,127.9,127.5,27.7,24.8,22.5,18.1,16.7$, 7.4. Anal Calcd for $\mathrm{C}_{27} \mathrm{H}_{30} \mathrm{OSi}$ : C, 81.35; H, 7.59. Found: C, 81.25; H, 7.50.

1b. Following the general procedure outlined above, cyclopropanation of allyl tertbutyldiphenylsilane with $n$-nutyl diazomethyl ketone afforded $\mathbf{1 b}($ trans $/$ cis $=2.1: 1)$ as a colorless liquid, $454 \mathrm{mg}, 60 \%$.

trans-1b. ${ }^{1} \mathrm{H}$ NMR (400 MHz, $\left.\mathrm{CDCl}_{3}\right): \delta$ 7.62-7.56 (4H, m), 7.41-7.31 (6H, m), 2.21-2.13 (1H, ddd, $J=16.4,8.3,6.6 \mathrm{~Hz}), 2.06-1.98$ (1H, ddd, $J=16.4,8.3,6.6 \mathrm{~Hz}), 1.52-1.48(1 \mathrm{H}$, m), 1.43-1.16 (7H, m), 1.11-1.07 (1H, m), $1.04(9 \mathrm{H}, \mathrm{s}), 0.84(3 \mathrm{H}, \mathrm{t}, J=7.3 \mathrm{~Hz}), 0.59-0.54$ $(1 \mathrm{H}, \mathrm{m}) .{ }^{13} \mathrm{C} \mathrm{NMR}\left(100 \mathrm{MHz}, \mathrm{CDCl}_{3}\right): \delta 210.3,136.0,135.9,134.6,134.2,129.1,129.08$, 127.6, 43.0, 30.9, 27.8, 25.9, 22.2, 21.5, 20.4, 18.0, 15.5, 13.8. Anal Calcd for $\mathrm{C}_{25} \mathrm{H}_{34} \mathrm{OSi}$ : , 79.31; H, 9.05. Found: C, 79.20; H, 8.90.

cis-1b. ${ }^{1} \mathrm{H}$ NMR (400 MHz, $\left.\mathrm{CDCl}_{3}\right):$ 8 7.66-7.59 (4H, m), 7.40-7.32 (6H, m), 2.43-2.35 (1H, ddd, $J=16.4,8.3,6.6 \mathrm{~Hz}), 2.28-2.20$ (1H, ddd, $J=16.4,8.3,6.6 \mathrm{~Hz}), 1.91-1.86(1 \mathrm{H}, \mathrm{m})$, 1.57-1.22 (8H, m), $1.03(9 \mathrm{H}, \mathrm{s}), 0.90(3 \mathrm{H}, \mathrm{t}, J=7.3 \mathrm{~Hz}), 0.81-0.76(1 \mathrm{H}, \mathrm{m}) .{ }^{13} \mathrm{C}$ NMR (100 $\left.\mathrm{MHz}, \mathrm{CDCl}_{3}\right): \delta 209.0,136.2,136.1,134.9,134.6,129.0,128.97,127.5,127.46,44.6,27.8$, 27.1, 26.1, 22.4, 21.4, 18.1, 16.9, 13.9, 7.2. Anal Calcd for $\mathrm{C}_{25} \mathrm{H}_{34} \mathrm{OSi}$ : C, 79.31; $\mathrm{H}, 9.05$. Found: C, 79.15; H, 8.90.

1c. Following the general procedure outlined above, cyclopropanation of allyl tertbutyldiphenylsilane with $t$-butyl diazomethyl ketone afforded 1c (trans/cis $=2: 1$ ) as a colorless liquid, $454 \mathrm{mg}, 60 \%$.

trans-1c. ${ }^{1} \mathrm{H}$ NMR (400 MHz, $\left.\mathrm{CDCl}_{3}\right): \delta$ 7.64-7.58 (4H, m), 7.39-7.32 (6H, m), 1.89-1.85 $(1 \mathrm{H}, \mathrm{m}), 1.59-1.54(1 \mathrm{H}, \mathrm{dd}, J=14.9,4.6 \mathrm{~Hz}), 1.40-1.33(2 \mathrm{H}, \mathrm{m}), 1.27-1.21(1 \mathrm{H}, \mathrm{m}), 1.07$ $(9 \mathrm{H}, \mathrm{s}), 1.06(9 \mathrm{H}, \mathrm{s}), 0.57-0.53(1 \mathrm{H}, \mathrm{m}) .{ }^{13} \mathrm{C} \mathrm{NMR}\left(100 \mathrm{MHz}, \mathrm{CDCl}_{3}\right): \delta 214.5,136.0,135.9$, 134.5, 134.47, 129.1, 127.6, 127.5, 43.6, 27.8, 26.8, 26.2, 21.6, 21.0, 18.0, 15.7. Anal Calcd for $\mathrm{C}_{25} \mathrm{H}_{34} \mathrm{OSi}$ : C, 79.31; H, 9.05. Found: C, 79.23; H, 8.94. 
cis-1c. ${ }^{1} \mathrm{H}$ NMR (400 MHz, $\left.\mathrm{CDCl}_{3}\right)$ : $\delta$ 7.68-7.66 (2H, m), 7.63-7.60 (2H, m), 7.41-7.32 (6H, m), 2.21-2.16 (1H, m), 1.37-1.25 (2H, m), 1.21-1.02 (1H, m), $1.17(9 \mathrm{H}, \mathrm{s}), 1.04(9 \mathrm{H}, \mathrm{s}), 0.95-$ $0.88(1 \mathrm{H}, \mathrm{m}), 0.76-0.71(1 \mathrm{H}, \mathrm{m}) .{ }^{13} \mathrm{C} \mathrm{NMR}\left(100 \mathrm{MHz}, \mathrm{CDCl}_{3}\right): \delta 213.3,136.2,136.0,134.9$, 134.6, 129.0, 128.97, 127.5, 127.4, 44.2, 27.8, 26.3, 23.1, 21.5, 18.1, 16.5, 7.3. Anal Calcd for $\mathrm{C}_{25} \mathrm{H}_{34} \mathrm{OSi}$ : C, 79.31; H, 9.05. Found: C, 79.16; H, 8.94.

16. Following the general procedure outlined above, cyclopropanation of allyl tertbutyldiphenylsilane with 2-diazocyclohexanone afforded 16 (trans/cis $=3: 1)$ as a colorless liquid, $226 \mathrm{mg}, 30 \%$.

trans-16. ${ }^{1} \mathrm{H}$ NMR $\left(400 \mathrm{MHz}, \mathrm{CDCl}_{3}\right): \delta$ 7.62-7.56 (4H, m), 7.40-7.32 (6H, m), 2.30-2.15 $(2 \mathrm{H}, \mathrm{m}), 1.78-1.64(7 \mathrm{H}, \mathrm{m}), 1.49-1.33(3 \mathrm{H}, \mathrm{m}), 1.07(9 \mathrm{H}, \mathrm{s}), 0.09-0.07(1 \mathrm{H}, \mathrm{m}) .{ }^{13} \mathrm{C} \mathrm{NMR}$ $\left(100 \mathrm{MHz}, \mathrm{CDCl}_{3}\right): \delta 211.5,136.0,135.9,134.5,134.4,129.1,127.6,127.5,39.7,33.6,28.1$, 27.9, 26.1, 26.0, 23.8, 23.6, 18.2, 10.0. Anal Calcd for $\mathrm{C}_{25} \mathrm{H}_{32} \mathrm{OSi}$ : C, 79.73; H, 8.56. Found: C, 79.60; H, 8.45.

cis-16. ${ }^{1} \mathrm{H}$ NMR $\left(400 \mathrm{MHz}, \mathrm{CDCl}_{3}\right): \delta$ 7.66-7.56 (4H, m), 7.40-7.32 (6H, m), 2.04-1.88 (2H, m), 1.76-1.65 (3H, m), 1.25-0.98 (7H, m), $1.02(9 \mathrm{H}, \mathrm{s}), 0.41-0.35(1 \mathrm{H}, \mathrm{m}) .{ }^{13} \mathrm{C}$ NMR $(100$ $\left.\mathrm{MHz}, \mathrm{CDCl}_{3}\right): \delta 209.8,136.1,136.0,134.8,134.4,129.0,127.6,127.5,42.0,36.0,35.8,29.3$, 27.8, 25.1, 23.9, 20.3, 18.1, 8.6. Anal Calcd for $\mathrm{C}_{25} \mathrm{H}_{32} \mathrm{OSi}$ : C, 79.73; H, 8.56. Found: C, $79.56 ; \mathrm{H}, 8.46$.

20. Following the general procedure outlined above, cyclopropanation of allyl tertbutyldiphenylsilane with 2-diazocyclopentanone afforded $\mathbf{2 0}($ trans $/$ cis $=1.1: 1)$ as a colorless liquid, $232 \mathrm{mg}, 32 \%$.

trans-20 (colorless dense liquid). ${ }^{1} \mathrm{H}$ NMR: $\delta$ 7.61-7.55 (4H, m), 7.40-7.31 (6H, m), 2.24$2.16(1 \mathrm{H}, \mathrm{m}), 2.12-2.04(1 \mathrm{H}, \mathrm{m}), 1.94-1.86(2 \mathrm{H}, \mathrm{m}), 1.79-1.72(2 \mathrm{H}, \mathrm{m}), 1.47-1.41(1 \mathrm{H}, \mathrm{m})$, 1.38-1.33 (1H, dd, $J=14.6,4.9 \mathrm{~Hz}), 1.21-1.18(1 \mathrm{H}, \mathrm{m}), 1.07(9 \mathrm{H}, \mathrm{s}), 0.97-0.94(1 \mathrm{H}, \mathrm{dd}, J=$ 14.6, $9.0 \mathrm{~Hz}), 0.34-0.31(1 \mathrm{H}, \mathrm{m}) .{ }^{13} \mathrm{C}$ NMR: $\delta 219.3,136.0,135.9,134.3,129.1,127.6$, 127.5, 38.7, 35.3, 27.9, 27.8, 27.3, 25.4, 20.7, 18.1, 11.4. Anal. Calcd. for $\mathrm{C}_{24} \mathrm{H}_{30} \mathrm{OSi}$ : C, 79.50; H, 8.34. Found: C, 79.38; H, 8.24.

cis-20 (colorless dense liquid). ${ }^{1} \mathrm{H}$ NMR: $\delta$ 7.66-7.57 (4H, m), 7.42-7.31 (6H, m), 2.18-2.09 $(1 \mathrm{H}, \mathrm{m}), 2.03-1.95(1 \mathrm{H}, \mathrm{m}), 1.82-1.75(2 \mathrm{H}, \mathrm{m}), 1.61-1.50(4 \mathrm{H}, \mathrm{m}), 1.30-1.23(1 \mathrm{H}, \mathrm{m}), 1.01$ 
(9H, s), 0.99-0.91 (2H, m). ${ }^{13} \mathrm{C}$ NMR: $\delta 218.5,136.2,136.1,134.7,134.5,129.0,127.53$, 127.5, 40.1, 34.7, 34.0, 30.0, 27.8, 25.6, 20.7, 18.1, 7.6. Anal. Calcd. for $\mathrm{C}_{24} \mathrm{H}_{30} \mathrm{OSi}$ : C, 79.50; H, 8.34. Found: C, 79.35; H, 8.25.

Preparation of tert-Butyldiphenylsillylallene 2c: An oven-dried $50 \mathrm{~mL}$ round bottom flask was charged with $1.712 \mathrm{~g}(6.53 \mathrm{mmol})$ of $\mathrm{Ph}_{3} \mathrm{P}$ under nitrogen atmosphere and $14 \mathrm{~mL}$ of THF was added. The solution was cooled to $-15{ }^{\circ} \mathrm{C}$ and a solution of $1.264 \mathrm{~g}(6.26 \mathrm{mmol})$ of diisopropylazodicarboxylate in $2 \mathrm{~mL}$ THF was added over $2 \mathrm{~min}$, followed immediately by the addition of a solution of $1.6 \mathrm{~g}(5.44 \mathrm{mmol})$ of 3-tert-butyldiphenylsilyl-2-propyn-1-ol in 4 $\mathrm{mL}$ THF over $2 \mathrm{~min}$. After stirring the contents for $5 \mathrm{~min}$, a solution of $1.77 \mathrm{~g}(6.53 \mathrm{mmol})$ of $o$-nitrobenznesulfonyl hydrazide in $8 \mathrm{~mL}$ THF was added to the reaction mixture over $5 \mathrm{~min}$. The resultant orange-red solution was stirred at $-15^{\circ} \mathrm{C}$ for $45 \mathrm{~min}$, allowed to warm up to 25 ${ }^{\circ} \mathrm{C}$ and held at that temperature for $5 \mathrm{~h}$. The reaction mixture was poured into a separatory funnel containing $50 \mathrm{~mL}$ hexane and washed with water $(4 \mathrm{x} 10 \mathrm{~mL})$ and brine $(1 \mathrm{x} 10 \mathrm{~mL})$. The organic layer was dried and concentrated. Purification of the residue by column chromatography gave the pure product, $912 \mathrm{mg}, 61 \%$, colorless liquid. ${ }^{1} \mathrm{H}$ NMR: $\delta$ 7.66-7.64 (4H, m), 7.40-7.32 (6H, m), 5.31-5.27 (1H, dd, $J=7.3,7.3 \mathrm{~Hz}), 4.38(2 \mathrm{H}, \mathrm{d}, J=7.3 \mathrm{~Hz})$, 1.09 (9H, s). ${ }^{13} \mathrm{C}$ NMR: $\delta 215.1,135.9,134.2,129.2,127.5,75.8,68.0,27.4,18.9$. Anal. Calcd. for $\mathrm{C}_{19} \mathrm{H}_{22} \mathrm{Si}$ : C, 81.95; H, 7.96. Found: C, 81.80; H, 7.84.

General procedure for the $\mathbf{T i C l}_{4}$-assisted reaction. A solution of freshly distilled $\mathrm{TiCl}_{4}(61$ $\mathrm{mg}, 0.326 \mathrm{mmol})$ in anhydrous $\mathrm{CH}_{2} \mathrm{Cl}_{2}(0.5 \mathrm{~mL})$ was added slowly under $\mathrm{N}_{2}$ to the stirred solution of a cylopropylmethylsilane $(0.251 \mathrm{mmol})$ and an allenylsilane $(0.326 \mathrm{mmol})$ in anhydrous $\mathrm{CH}_{2} \mathrm{Cl}_{2}(0.8 \mathrm{~mL})$ at $-78{ }^{\circ} \mathrm{C}$. The reaction mixture turned deep red. After stirring for $3 \mathrm{~h}$ at $-78{ }^{\circ} \mathrm{C}$, it was warmed slowly over $1 \mathrm{~h}$ to $-40{ }^{\circ} \mathrm{C}$. The stirring was continued at this temperature for $2 \mathrm{~h}$ and the reaction mixture was taken in $\mathrm{Et}_{2} \mathrm{O}(10 \mathrm{~mL})$. The ethereal solution was washed with water $(1 \mathrm{x} 7 \mathrm{~mL})$. The aqueous washing was extracted with $\mathrm{Et}_{2} \mathrm{O}(2$ x $5 \mathrm{~mL}$ ). The combined organic extracts were washed with brine, dried, and concentrated. Purification of the residue by silica gel column chromatography (EtOAc/hexanes) furnished the pure product(s). The separation of the cis- and trans-isomers was achieved by radial chromatography over silica gel (EtOAc/hexanes). 
General procedure for the $\mathbf{E t}_{2} \mathbf{A l C l}$-assisted reaction. A solution of $\mathrm{Et}_{2} \mathrm{AlCl}$ ( $54 \mathrm{mg}, 0.375$ mmol) in toluene $(208 \mu \mathrm{L})$ was added slowly under $\mathrm{N}_{2}$ to the stirred solution of a cylopropylmethylsilane $(0.251 \mathrm{mmol})$ and an allenylsilane $(0.326 \mathrm{mmol})$ in anhydrous $\mathrm{CH}_{2} \mathrm{Cl}_{2}(1.3 \mathrm{~mL})$ at $25^{\circ} \mathrm{C}$ when the reaction mixture turned pale yellow. After stirring for $6 \mathrm{~h}$, the reaction mixture was quenched with water at $0{ }^{\circ} \mathrm{C}$ and diluted with $\mathrm{Et}_{2} \mathrm{O}(10 \mathrm{~mL})$. The ethereal solution was washed with water $(7 \mathrm{~mL})$. The aqueous washing was extracted with $\mathrm{Et}_{2} \mathrm{O}(2 \times 5 \mathrm{~mL})$. The combined organic extracts were washed with brine, dried, and concentrated. Purification of the residue by silica gel column chromatography (EtOAc/hexanes) furnished the pure product.

5. Following the general procedure for the $\mathrm{TiCl}_{4}$-assisted reaction outlined above, reaction of cylopropylmethylsilane 1a with the allenylsilane $\mathbf{2 a}$ afforded $5($ cis/trans $=3.2: 1)$ as a colorless liquid, $102 \mathrm{mg}, 90 \%$.

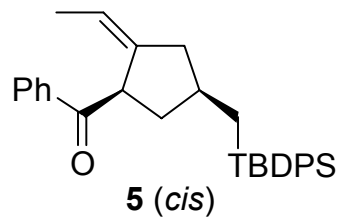

${ }^{1} \mathrm{H}$ NMR: $\delta$ 7.87-7.85 (2H, m), 7.65-7.60 (4H, m), 7.55-7.52 $(1 \mathrm{H}, \mathrm{m}), 7.44-7.31(8 \mathrm{H}, \mathrm{m})$, 5.33-5.27 $(1 \mathrm{H}, \mathrm{m}), 4.14-4.10(1 \mathrm{H}, \mathrm{m}), 2.13-1.93(4 \mathrm{H}, \mathrm{m}), 1.35-1.29(3 \mathrm{H}, \mathrm{m}), 1.25(3 \mathrm{H}, \mathrm{d}, J=$ $6.3 \mathrm{~Hz}) .0 .99$ (9H, s). ${ }^{13} \mathrm{C}$ NMR: $\delta$ 201.6, 142.4, 136.8, 136.1, 136.0, 134.9, 134.8, 132.8, 129.1, 129.0, 128.5, 128.4, 127.5, 117.3, 49.0, 45.1, 43.0, 36.2, 27.8, 18.1, 15.7, 14.9. Anal. Calcd. for $\mathrm{C}_{31} \mathrm{H}_{36} \mathrm{OSi}$ : C, 82.25; H, 8.02. Found: C, 82.14; H, 7.94.

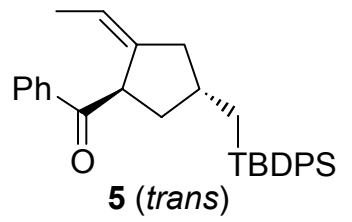

${ }^{1} \mathrm{H}$ NMR: $\delta$ 7.78-7.76 (2H, m), 7.60-7.50 (5H, m), 7.41-7.26 $(8 \mathrm{H}, \mathrm{m}), 5.45-5.40(1 \mathrm{H}, \mathrm{m})$, 4.29-4.25 (1H, m), 2.46-2.39 (1H, m), 2.27-2.20 (1H, m), 1.93-1.87 (1H, m), 1.71-1.63 (1H, m), 1.60-1.53 (1H, m), 1.37-1.35 (3H, dd, $J=6.8,1.0 \mathrm{~Hz}), 1.30-1.25(1 \mathrm{H}, \mathrm{dd}, J=15.1,6.6$ $\mathrm{Hz}), 1.20-1.14(1 \mathrm{H}, \mathrm{dd}, J=15.1,7.8 \mathrm{~Hz}), 1.01(9 \mathrm{H}, \mathrm{s}) .{ }^{13} \mathrm{C} \mathrm{NMR}: \delta 200.9,141.7,136.5$, $136.0,135.96,135.2$, 134.9, 132.6, 129.0, 128.97, 128.4, 128.38, 127.5, 118.4, 47.9, 43.8, 
41.2, 34.2, 27.9, 18.1, 15.7, 15.4. Anal. Calcd. for $\mathrm{C}_{31} \mathrm{H}_{36} \mathrm{OSi}$ : C, 82.25; H, 8.02. Found: C, $82.15 ; \mathrm{H}, 7.90$.

6. Following the general procedure for the $\mathrm{TiCl}_{4}$-assisted reaction outlined above, reaction of the cylopropylmethylsilane 1a with the allenylsilane 2a in presence of 1.3 equiv of $\mathrm{Et}_{2} \mathrm{AlCl}$ afforded $\mathbf{6}$ (cis/trans $=7: 1)$ as a colorless liquid, $76 \mathrm{mg}, 58 \%$.

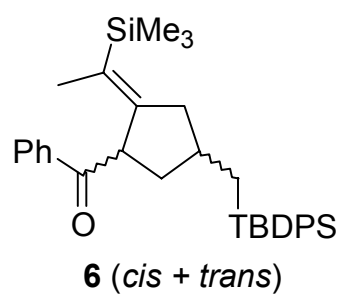

Mixture of the trans- and cis-isomers. ${ }^{1} \mathrm{H}$ NMR: 7.91-7.88 (m), 7.81-7.78 (m), 7.74-7.52 (m), 7.46-7.30 (m), 4.39-4.36 (m), 4.28-4.24 (m), 2.31-2.14 (m), 1.97-1.94 (m), 1.64-1.55 (m), 1.43-1.24 (m), $1.38(\mathrm{~s}), 1.29(\mathrm{~s}), 1.02(\mathrm{~s}), 1.01(\mathrm{~s}), 0.01(\mathrm{~s}),-0.1(\mathrm{~s}) .{ }^{13} \mathrm{C}$ NMR: $\delta 201.7,152.1$, $137.0,136.1,136.0,134.9,134.7,132.7,132.6,129.1,129.0,128.5,128.4,128.3,127.6$, $127.58,127.4,51.8,51.0,44.9,44.0,43.1,40.6,27.9,27.0,19.6,18.1,16.0,15.9,-0.6,-0.63$. Anal. Calcd. for $\mathrm{C}_{34} \mathrm{H}_{44} \mathrm{OSi}_{2}$ : C, 77.80; H, 8.45. Found: C, 77.67; H, 8.35.

7. Following the general procedure for the $\mathrm{Et}_{2} \mathrm{AlCl}$-assisted reaction outlined above, reaction of the cylopropylmethylsilane 1a with the allenylsilane $\mathbf{2 a}$ afforded 7 as a colorless liquid, 85 $\mathrm{mg}, 65 \%$.

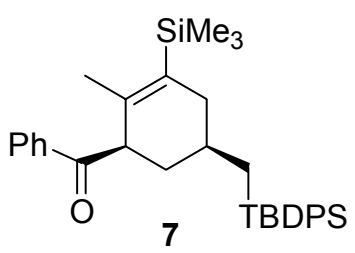

${ }^{1} \mathrm{H}$ NMR: $\delta$ 7.94-7.91 (2H, m), 7.54-7.52 (4H, m), 7.45-7.29 (9H, m), $4.08(1 \mathrm{H}, \mathrm{d}, J=5.6$ Hz), 2.75-2.68 (1H, tdd, $J=15.8,8.1,1.9 \mathrm{~Hz}), 2.55-2.47(1 \mathrm{H}, \mathrm{m}), 2.14-2.07(1 \mathrm{H}, \mathrm{m}), 1.63$ $(3 \mathrm{H}, \mathrm{s}), 1.49-1.41(2 \mathrm{H}, \mathrm{m}), 1.14-1.01(2 \mathrm{H}, \mathrm{m}), 0.94(9 \mathrm{H}, \mathrm{s}), 0.13(9 \mathrm{H}, \mathrm{s}) .{ }^{13} \mathrm{C}$ NMR: $\delta 203.2$, $146.5,137.9,137.8,135.8,134.7,134.6,132.8,128.9,128.5,128.4,127.5,66.7,47.9,43.1$, 30.2, 27.8, 18.0, 16.5, 9.0, -0.6. Anal. Calcd. for $\mathrm{C}_{34} \mathrm{H}_{44} \mathrm{OSi}_{2}$ : C, 77.80; $\mathrm{H}, 8.45$. Found: $\mathrm{C}$, 77.64; H, 8.36. 
8, 9. Following the general procedure for the $\mathrm{TiCl}_{4}$-assisted reaction outlined above, reaction of the cylopropylmethylsilane $\mathbf{1 a}$ with the allenylsilane $\mathbf{2 b}$ afforded a mixture of $\mathbf{8}$ and $\mathbf{9}$ ( $\mathbf{8} / \mathbf{9}$ $=1.7: 1), 147 \mathrm{mg}, 85 \%$.

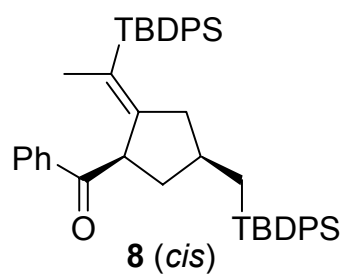

${ }^{1} \mathrm{H}$ NMR: $\delta$ 7.86-7.83 $(2 \mathrm{H}, \mathrm{m}), 7.73-7.71(2 \mathrm{H}, \mathrm{m}), 7.58-7.51(3 \mathrm{H}, \mathrm{m}), 7.46-7.20(18 \mathrm{H}, \mathrm{m})$, 4.36-4.31 (1H, m), 2.06-2.01 (1H, m), 1.83-1.67 (1H, m), $1.64(3 \mathrm{H}, \mathrm{s}), 1.57-1.50(2 \mathrm{H}, \mathrm{m})$, 1.32-1.24 (2H, m), 1.08-1.02 (1H, m), $1.00(9 \mathrm{H}, \mathrm{s}), 0.83(9 \mathrm{H}, \mathrm{s}) .{ }^{13} \mathrm{C}$ NMR: $\delta 201.1,157.2$, $136.7,136.1,136.0,135.9,135.8,135.7,135.57,135.1,134.2,132.7,128.84,128.8,128.7$, $128.56,128.5,128.3,127.5,127.4,52.5,47.4,41.5,36.1,28.6,27.7,21.9,19.1,17.9,15.7$. Anal. Calcd. for $\mathrm{C}_{47} \mathrm{H}_{54} \mathrm{OSi}_{2}$ : C, 81.68; H, 7.88. Found: $\mathrm{C}, 81.55 ; \mathrm{H}, 7.75$.

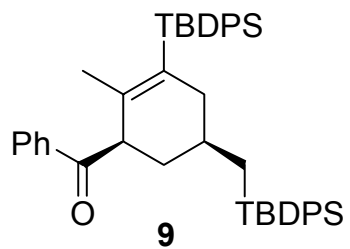

${ }^{1} \mathrm{H}$ NMR: $\delta$ 7.92-7.90 (2H, m), 7.72-7.71 (2H, m), 7.62-7.53 (7H, m), 7.44-7.30 (14H, m), $4.08(1 \mathrm{H}$, broad s), 3.00-2.98 $(1 \mathrm{H}, \mathrm{m}), 2.56-2.47(2 \mathrm{H}, \mathrm{m}), 1.63-1.52(4 \mathrm{H}, \mathrm{m}), 1.20(3 \mathrm{H}, \mathrm{s})$, $1.10(9 \mathrm{H}, \mathrm{s}), 0.97$ (9H, s). ${ }^{13} \mathrm{C}$ NMR: $\delta 202.2,151.9,137.5,136.1,135.9,135.8,135.6,135.1$, $134.52,134.5,132.9,132.8,129.0,128.9,128.8,128.6,128.4,127.63,127.6,66.5,47.4$, 45.1, 30.4, 28.2, 27.8, 19.0, 18.4, 18.1, 8.6. Anal. Calcd. for $\mathrm{C}_{47} \mathrm{H}_{54} \mathrm{OSi}_{2}$ : C, 81.68; H, 7.88. Found: C, 81.50; H, 7.74.

10. Following the general procedure for the $\mathrm{TiCl}_{4}$-assisted reaction outlined above, reaction of the cylopropylmethylsilane $\mathbf{1 b}$ with the allenylsilane $\mathbf{2 a}$ afforded $\mathbf{1 0}($ cis/trans $=1.9: 1)$ as a colorless liquid, $82 \mathrm{mg}, 75 \%$. 


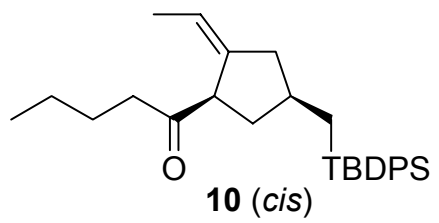

${ }^{1} \mathrm{H}$ NMR: $\delta$ 7.63-7.61 (4H, m), 7.40-7.33 (6H, m), 5.27-5.22 (1H, m), 3.28-3.23 (1H, m), 2.34-2.29 (2H, m), 2.07-2.03 (1H, m), 1.90-1.85 (2H, m), 1.53-1.44 (2H, m), $1.35(3 \mathrm{H}, \mathrm{d}, J=$ $6.6 \mathrm{~Hz}), 1.32-1.20(6 \mathrm{H}, \mathrm{m}), 1.00(9 \mathrm{H}, \mathrm{s}), 0.88(3 \mathrm{H}, \mathrm{t}, J=7.3 \mathrm{~Hz}) .{ }^{13} \mathrm{C} \mathrm{NMR}: \delta 212.1,141.6$, 136.0, 134.8, 129.1, 127.5, 118.1, 54.5, 45.3, 40.6, 39.3, 35.7, 27.8, 26.0, 22.4, 18.1, 15.8, 14.4, 13.9. Anal. Calcd. for $\mathrm{C}_{29} \mathrm{H}_{40} \mathrm{OSi}$ : C, 80.49; H, 9.32. Found: C, 80.35; H, 9.24.

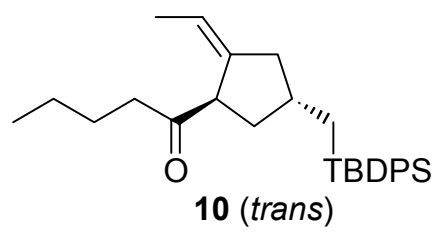

${ }^{1} \mathrm{H}$ NMR: $\delta$ 7.61-7.59 (4H, m), 7.41-7.33 (6H, m), 5.36-5.30 (1H, m), 2.34-2.29 (2H, m), 2.22-2.10 (1H, m), 1.90-1.79 (2H, m), 1.50-1.41 (5H, m), 1.39 (3H, d, $J=6.6 \mathrm{~Hz}), 1.24-1.10$ $(4 \mathrm{H}, \mathrm{m}), 1.00(9 \mathrm{H}, \mathrm{s}), 0.83(3 \mathrm{H}, \mathrm{t}, J=7.3 \mathrm{~Hz}) .{ }^{13} \mathrm{C}$ NMR: $\delta 212.1,141.1,136.0,134.9,129.0$, 127.5, 118.9, 53.6, 43.4, 39.8, 39.6, 34.1, 27.8, 25.8, 22.3, 18.1, 15.7, 15.0, 13.9. Anal. Calcd. for $\mathrm{C}_{29} \mathrm{H}_{40} \mathrm{OSi}$ : C, 80.49; H, 9.32. Found: C, 80.38; H, 9.22.

11. Following the general procedure for the $\mathrm{Et}_{2} \mathrm{AlCl}$-assisted reaction outlined above, reaction of the cylopropylmethylsilane $\mathbf{1 b}$ with the allenylsilane $\mathbf{2 a}$ afforded $\mathbf{1 1}$ as a colorless liquid, $82 \mathrm{mg}, 65 \%$.

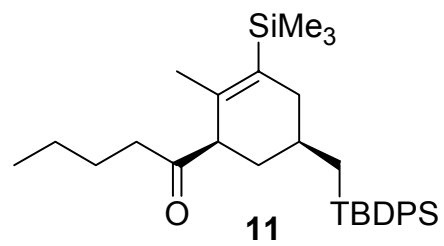

${ }^{1} \mathrm{H}$ NMR: $\delta$ 7.60-7.56 (4H, m), 7.39-7.32 (6H, m), 3.14-3.12 (1H, m), 2.73-2.65 (1H, tdd, $J=$ 16.1, 8.0, $2.2 \mathrm{~Hz}), 2.36-2.30(2 \mathrm{H}, \mathrm{m}), 2.28-2.24(1 \mathrm{H}, \mathrm{m}), 2.09-2.04(1 \mathrm{H}, \mathrm{m}), 1.64(3 \mathrm{H}, \mathrm{s})$, 1.54-1.47 (2H, m), 1.41-1.22 (4H, s), 1.12-1.05 (2H, m), $1.02(9 \mathrm{H}, \mathrm{s}), 0.88(3 \mathrm{H}, \mathrm{t}, J=7.3$ $\mathrm{Hz}), 0.11(9 \mathrm{H}, \mathrm{s}) .{ }^{13} \mathrm{C}$ NMR: $\delta$ 213.2, 145.7, 137.0, 135.9, 135.8, 134.7, 134.6, 128.9, 127.6, 
72.9, 45.6, 43.6, 40.2, 30.5, 27.8, 25.8, 22.4, 18.1, 16.2, 13.9, 8.5, -0.7. Anal. Calcd. for $\mathrm{C}_{32} \mathrm{H}_{48} \mathrm{OSi}_{2}$ : C, 76.12; H, 9.58. Found: C, 75.95; H, 9.45.

12. Following the general procedure for the $\mathrm{Et}_{2} \mathrm{AlCl}$-assisted reaction outlined above, reaction of the cylopropylmethylsilane $\mathbf{1 b}$ with the allenylsilane $\mathbf{2 b}$ afforded $\mathbf{1 2}$ as a colorless liquid, $93 \mathrm{mg}, 55 \%$.

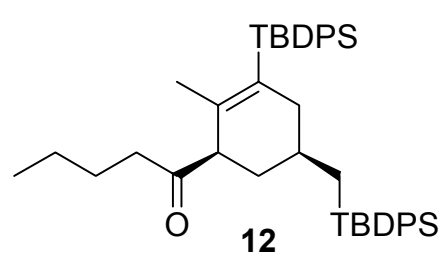

${ }^{1} \mathrm{H}$ NMR: $\delta$ 7.62-7.54 (8H, m), 7.40-7.25 (12H, m), 3.19 (1H, broad s), 3.03-2.97 (1H, tdd, $J$ $=16.1,8.0,2.1 \mathrm{~Hz}), 2.45-2.31(4 \mathrm{H}, \mathrm{m}), 1.56-1.40(5 \mathrm{H}, \mathrm{m}), 1.33-1.24(2 \mathrm{H}$, sextet, $J=7.3 \mathrm{~Hz})$, $1.19(3 \mathrm{H}, \mathrm{s}), 1.17-1.12(1 \mathrm{H}, \mathrm{m}), 1.07(9 \mathrm{H}, \mathrm{s}), 1.03(9 \mathrm{H}, \mathrm{s}), 0.89(3 \mathrm{H}, \mathrm{t}, J=7.3 \mathrm{~Hz}) .{ }^{13} \mathrm{C} \mathrm{NMR}$ : $\delta 212.4,151.1,137.0,135.9,135.8,135.3,134.6,129.0,128.9,127.6,72.6,46.0,45.4,40.5$, 30.8, 28.2, 27.9, 25.8, 22.5, 19.0, 18.1, 18.15, 13.9, 8.2. Anal. Calcd. for $\mathrm{C}_{45} \mathrm{H}_{58} \mathrm{OSi}_{2}: \mathrm{C}$, 80.54; H, 8.71. Found: C, 80.44; H, 8.60.

13. Following the general procedure for the $\mathrm{TiCl}_{4}$-assisted reaction outlined above, reaction of the cylopropylmethylsilane $\mathbf{1 c}$ with the allenylsilane $\mathbf{2 b}$ afforded $\mathbf{1 3}$ as a colorless liquid, $109 \mathrm{mg}, 65 \%$.

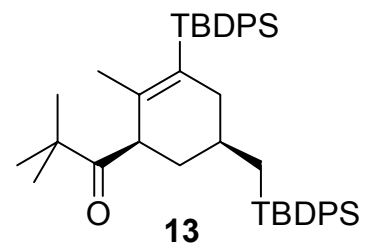

${ }^{1} \mathrm{H}$ NMR: $\delta$ 7.75-7.73 (2H, m), 7.61-7.58 (7H, m), 7.39-7.25 $(11 \mathrm{H}, \mathrm{m}), 3.50(1 \mathrm{H}$, broad s), 2.98-2.92 (1H, tdd, $J=15.6,7.6,2.2 \mathrm{~Hz}), 2.53-2.50$ (1H, m), 2.13-2.11 (1H, m), 1.53-1.39 $(2 \mathrm{H}, \mathrm{m}), 1.30-1.22(2 \mathrm{H}, \mathrm{m}), 1.12(3 \mathrm{H}, \mathrm{s}), 1.10(9 \mathrm{H}, \mathrm{s}), 1.07(9 \mathrm{H}, \mathrm{s}), 1.02(9 \mathrm{H}, \mathrm{s}) .{ }^{13} \mathrm{C} \mathrm{NMR}: \delta$ $216.4,152.2$, 136.2, 135.9, 135.8, 135.2, 134.6, 132.8, 129.0, 128.8, 128.7, 127.7, 127.6, $127.5,65.5,47.0,44.7,44.6,30.6,28.2,27.9,26.1,18.9,18.3,18.2,8.0$. Anal. Calcd. for $\mathrm{C}_{45} \mathrm{H}_{58} \mathrm{OSi}_{2}: \mathrm{C}, 80.54 ; \mathrm{H}, 8.71$. Found: $\mathrm{C}, 80.45 ; \mathrm{H}, 8.63$. 
14. Following the general procedure for the $\mathrm{TiCl}_{4}$-assisted reaction outlined above, reaction of the cylopropylmethylsilane $1 \mathrm{a}$ with the allenylsilane $\mathbf{2} \mathbf{c}$ afforded $\mathbf{1 4}$ (cis/trans $=2.8: 1)$ as a colorless liquid, $105 \mathrm{mg}, 62 \%$.

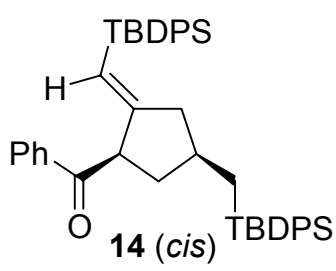

${ }^{1} \mathrm{H}$ NMR: $\delta$ 7.94-7.91 (2H, m), 7.55-7.21 (21H, m), 7.14-7.10 (2H, m), $5.46(1 \mathrm{H}, \mathrm{s}), 4.33-$ $4.28(1 \mathrm{H}, \mathrm{m}), 1.83-1.53(5 \mathrm{H}, \mathrm{m}), 1.21-1.07(2 \mathrm{H}, \mathrm{m}), 0.87(9 \mathrm{H}, \mathrm{s}), 0.75(9 \mathrm{H}, \mathrm{s}) .{ }^{13} \mathrm{C}$ NMR: $\delta$ $200.9,165.0,137.8,136.0,135.97,135.9,135.8,134.8,134.6,134.4,134.0,132.8,129.0$, $128.9,128.8,128.5,127.5,127.4,127.3,116.1,55.1,44.5,39.3,35.6,27.8,27.1,18.0,16.0$. Anal. Calcd. for $\mathrm{C}_{46} \mathrm{H}_{52} \mathrm{OSi}_{2}$ : C, 81.60; H, 7.74. Found: C, 81.47; H, 7.65.

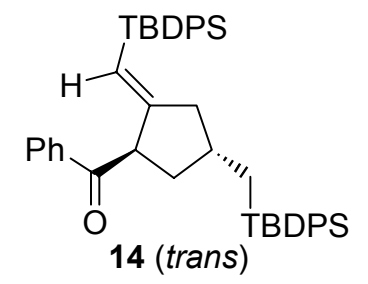

${ }^{1} \mathrm{H}$ NMR: $\delta{ }^{1} \mathrm{H}$ NMR: $\delta$ 7.85-7.83 (2H, m), 7.64-7.62 (2H, m), 7.55-7.21 (19H, m), 7.18-7.10 $(2 \mathrm{H}, \mathrm{m}), 5.63(1 \mathrm{H}, \mathrm{bs}), 4.49-4.45(1 \mathrm{H}, \mathrm{m}), 1.93-1.50(5 \mathrm{H}, \mathrm{m}), 1.27-1.11(2 \mathrm{H}, \mathrm{m}), 0.93(9 \mathrm{H}$, s), $0.80(9 \mathrm{H}, \mathrm{s}) .{ }^{13} \mathrm{C}$ NMR: $\delta 200.9,164.7,137.5,136.0,135.97,135.9,135.7,134.8,134.6$, 134.4, 134.2, 132.6, 128.9, 128.87, 128.8, 128.4, 127.5, 127.4, 127.3, 117.2, 54.0, 44.7, 38.4, 34.6, 27.9, 27.1, 18.0, 17.99, 16.5. Anal. Calcd. for $\mathrm{C}_{46} \mathrm{H}_{52} \mathrm{OSi}_{2}$ : C, 81.60; H, 7.74. Found: C, $81.45 ; \mathrm{H}, 7.50$.

15. Following the general procedure for the $\mathrm{TiCl}_{4}$-assisted reaction outlined above, reaction of the cylopropylmethylsilane $1 \mathrm{c}$ with the allenylsilane $\mathbf{2 c}$ afforded 15 (cis/trans $=5.8: 1)$ as a colorless liquid, $99 \mathrm{mg}, 60 \%$. 


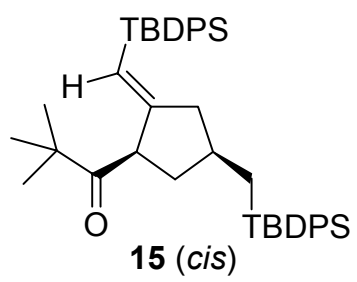

${ }^{1} \mathrm{H}$ NMR: $\delta$ 7.65-7.63 (2H, m), 7.50-7.20 (16H, m), 7.13-7.09 (2H, m), 5.43-5.37 (1H, s), 3.88-3.83 (1H, m), 1.80-1.57 (4H, m), 1.32-1.06 (3H, m), $1.10(9 \mathrm{H}, \mathrm{s}), 0.87(9 \mathrm{H}, \mathrm{s}), 0.86(9 \mathrm{H}$, s). ${ }^{13} \mathrm{C}$ NMR: $\delta 218.0,167.7,136.1,135.9,135.8,135.7,134.9,134.8,134.2,133.9,128.8$, $128.7,127.5,127.4,127.3,54.3,45.2,44.6,42.6,36.1,27.7,27.2,26.9,18.0,17.9,15.9$. Anal. Calcd. for $\mathrm{C}_{44} \mathrm{H}_{56} \mathrm{OSi}_{2}$ : C, 80.43; H, 8.59. Found: C, 80.35; H, 8.50.

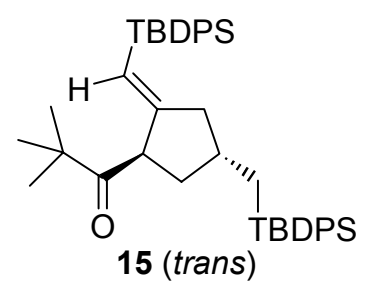

The trans-isomer could be fully separated from the $c i s$-isomer. However, it was contaminated with another product (uncharacterized) that it could not be separated from. Some characteristic ${ }^{1} \mathrm{H}$ absorptions for the trans-isomer are: $\delta 5.54(\mathrm{bs}), 4.05-4.02(\mathrm{~m})$.

17, 18. Following the general procedure for the $\mathrm{TiCl}_{4}$-assisted reaction outlined above, reaction of the cylopropylmethylsilane $\mathbf{1 6}$ with the allenylsilane $\mathbf{2 b}$ afforded a mixture of $\mathbf{1 7}$ $18(\mathbf{1 7} / \mathbf{1 8}=2: 1)$ as a colorless liquid, $151 \mathrm{mg}, 90 \%$.

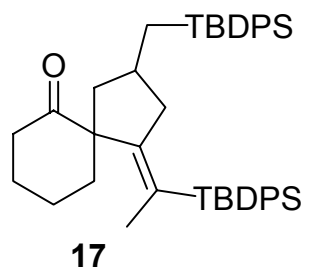

${ }^{1} \mathrm{H}$ NMR: $\delta$ 7.79-7.77 (2H, m), 7.59-7.57 (2H, m), 7.41-7.22 (16H, m), 2.41-2.37 (1H, m), 2.01-1.95 (1H, m), 1.85-1.71 (4H, m), $1.57(3 \mathrm{H}, \mathrm{s}), 1.45-1.39(3 \mathrm{H}, \mathrm{m}), 1.32-1.18(2 \mathrm{H}, \mathrm{m})$, $0.99(9 \mathrm{H}, \mathrm{s}), 0.92-0.74(4 \mathrm{H}, \mathrm{m}), 0.85(9 \mathrm{H}, \mathrm{s}) .{ }^{13} \mathrm{C}$ NMR: $\delta 212.5,161.0,136.7,136.3,136.0$, $135.9,135.5,135.3,134.4,128.9,128.8,128.6,128.5,127.6,127.4,122.4,61.9,47.5,47.2$, 
40.3, 34.6, 33.5, 28.5, 27.7, 24.5, 24.3, 22.2, 19.1, 17.9, 14.7. Anal. Calcd. for $\mathrm{C}_{45} \mathrm{H}_{56} \mathrm{OSi}_{2}$ : $\mathrm{C}$, 80.78; H, 8.44. Found: C, 80.65; H, 8.36.

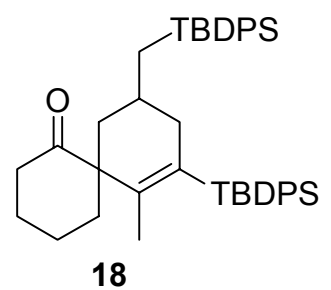

${ }^{1} \mathrm{H}$ NMR: $\delta$ 7.81-7.78 (2H, m), 7.63-7.58 (4H, m), 7.42-7.23 (14H, m), 2.57-2.31 (4H, m), 2.23-2.17 (1H, m), 1.88-1.82 (1H, m), 1.76-1.68 (1H, m), 1.62-1.48 (2H, m), 1.35-1.23 (3H, m), $1.11(3 \mathrm{H}, \mathrm{s}), 1.09(9 \mathrm{H}, \mathrm{s}), 1.03(9 \mathrm{H}, \mathrm{s}), 0.88-0.82(3 \mathrm{H}, \mathrm{m}) .{ }^{13} \mathrm{C}$ NMR: $\delta$ 213.0, 155.6, 136.0, 135.6, 135.2, 134.7, 134.4, 134.2, 130.2, 128.7, 128.5, 128.3, 127.3, 127.27, 127.1, $69.3,49.4,40.6,40.0,29.3,27.9,27.5,25.8,23.5,21.6,18.6,17.8,16.7,8.7$. Anal. Calcd. for $\mathrm{C}_{45} \mathrm{H}_{56} \mathrm{OSi}_{2}$ : C, 80.78; H, 8.44. Found: C, 80.65; H, 8.35.

19. Following the general procedure for the $\mathrm{TiCl}_{4}$-assisted reaction outlined above, reaction of the cylopropylmethylsilane $\mathbf{1 6}$ with the allenylsilane $\mathbf{2 c}$ afforded $\mathbf{1 9}$ as a colorless liquid, $90 \mathrm{mg}, 55 \%$.

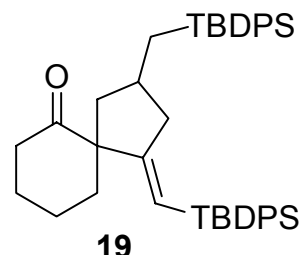

19

${ }^{1} \mathrm{H}$ NMR: $\delta$ 7.78-7.76 (2H, m), 7.56-7.54 (2H, m), 7.45-7.22 (14H, m), 7.20-7.15 (2H, m), $5.56(1 \mathrm{H}, \mathrm{bs}), 2.33-2.28(1 \mathrm{H}, \mathrm{m}), 2.18-2.10(1 \mathrm{H}, \mathrm{m}), 1.88-1.47(9 \mathrm{H}, \mathrm{m}), 1.34-1.26(1 \mathrm{H}, \mathrm{m})$, 1.11-0.97 (3H, m), 0.92 (9H, s), 0.87 (9H, s). ${ }^{13} \mathrm{C}$ NMR: $\delta 212.3,168.2,136.2,136.0,135.9$, $135.7,135.14,135.1,134.33,134.3,128.9,128.8,128.78,128.7,127.5,127.46,127.4,127.3$, 114.3, 63.0, 45.9, 45.3, 40.1, 38.3, 33.4, 27.7, 27.2, 26.2, 22.1, 18.1, 17.9, 15.6. Anal. Calcd. for $\mathrm{C}_{44} \mathrm{H}_{54} \mathrm{OSi}_{2}: \mathrm{C}, 80.67 ; \mathrm{H}, 8.31$. Found: $\mathrm{C}, 80.55 ; \mathrm{H}, 8.20$.

21, 22. Following the general procedure for the $\mathrm{TiCl}_{4}$-assisted reaction outlined above, reaction of the cylopropylmethylsilane $\mathbf{2 0}$ with the allenylsilane $\mathbf{2 b}$ afforded a mixture of $\mathbf{2 1}$ and $22(\mathbf{2 1} / \mathbf{2 2}=1: 1.1)$ as a colorless liquid, $148 \mathrm{mg}, 90 \%$. 


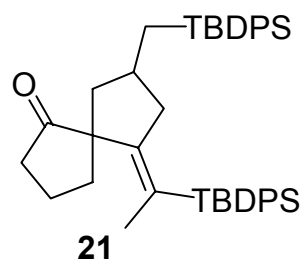

${ }^{1} \mathrm{H}$ NMR: $\delta$ 7.69-7.67 (2H, m), 7.56-7.54 (2H, m), 7.41-7.21 (16H, m), 2.43-2.31 (1H, m), 2.19-2.10 (1H, m), 2.04-1.86 (3H, m), $1.72(3 \mathrm{H}, \mathrm{bs}), 1.62-1.24(6 \mathrm{H}, \mathrm{m}), 1.13-1.01(1 \mathrm{H}, \mathrm{m})$, 0.99 (9H, s), 0.93-0.78 (1H, m), 0.85 (9H, s). ${ }^{13} \mathrm{C}$ NMR: $\delta 222.0,160.8,136.1,136.08,136.0$, $135.9,135.7,135.6,135.3,135.1,134.5,129.1,128.9,128.8,128.7,128.6,127.6,127.5$, 127.4, 123.0, 63.2, 47.4, 47.1, 37.4, 35.8, 34.5, 28.6, 27.7, 23.2, 19.8, 19.0, 17.9, 14.2. Anal. Calcd. for $\mathrm{C}_{44} \mathrm{H}_{54} \mathrm{OSi}_{2}:$ C, 80.67; H, 8.31. Found: C, 80.58; H, 8.22.

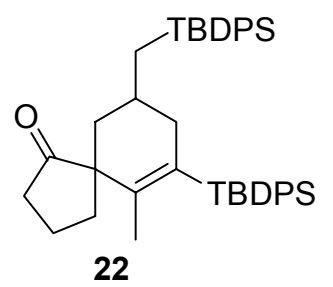

${ }^{1} \mathrm{H}$ NMR: $\delta$ 7.68-7.66 (2H, m), 7.59-7.55 (6H, m), 7.40-7.28 (12H, m), 2.90-2.84 (1H, m), 2.42-2.36 (1H, m), 2.31-2.06 (3H, m), 2.01-1.95 $(1 \mathrm{H}, \mathrm{m}), 1.85-1.78(1 \mathrm{H}, \mathrm{m}), 1.71-1.49(3 \mathrm{H}$, m), 1.39-1.12 (2H, m), $1.10(3 \mathrm{H}, \mathrm{bs}), 1.07(9 \mathrm{H}, \mathrm{s}), 1.01(9 \mathrm{H}, \mathrm{s}), 0.97-0.83(1 \mathrm{H}, \mathrm{m}) .{ }^{13} \mathrm{C} \mathrm{NMR}$ : $\delta 223.0,156.4,136.0,135.9,135.8,135.7,135.1,134.6,131.7,129.0,128.9,128.8,127.64$, 127.62, 127.6, 127.5, 69.0, 50.8, 43.0, 38.6, 28.2, 28.0, 27.9, 24.7, 19.8, 18.9, 18.1, 16.3, 9.1. Anal. Calcd. for $\mathrm{C}_{44} \mathrm{H}_{54} \mathrm{OSi}_{2}: \mathrm{C}, 80.67 ; \mathrm{H}, 8.31$. Found: $\mathrm{C}, 80.50 ; \mathrm{H}, 8.25$.

\section{References:}

1. Evans, D. A.; Sweeny, Z. K.; Rovis, T.; Tedrow, J. S. J. Am. Chem. Soc. 2001, 123, 12095. 